Disponível em

http://www.anpad.org.br/rac

RAC, Rio de Janeiro, v. 18, n. 5, art. 5, pp. 650-669, Set./Out. 2014

http://dx.doi.org/10.1590/1982-7849rac20141709

$($ (c) EY-NO

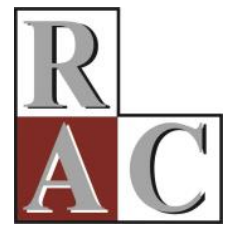

\title{
Perspectiva Institucional dos Sistemas de Informação em Saúde em Dois Estados Brasileiros
}

\section{An Institutional Perspective of Health Information Systems in two Brazilian States}

Vanessa Marques Daniel

E-mail: vanessa.daniel@ufrgs.br

Universidade Federal do Rio Grande do Sul - UFRGS

Rua Washington Luiz, 855, Centro Histórico, 90010-460, Porto Alegre, RS, Brasil.

Gabriela Viale Pereira

E-mail: gabiviale@ terra.com.br Pontifícia Universidade Católica do Rio Grande do Sul - PUCRS Av. Ipiranga, 6681, sala 1105, 90619-90, Porto Alegre, RS, Brasil.

Marie Anne Macadar E-mail: marie.macadar@pucrs.br Pontifícia Universidade Católica do Rio Grande do Sul - PUCRS Av. Ipiranga, 6681, sala 1105, 90619-90, Porto Alegre, RS, Brasil. 


\title{
Resumo
}

Os Sistemas de Informação em Saúde (SIS) são artefatos tecnológicos que respaldam gestores das três esferas governamentais a obter informações imprescindíveis para apoiarem a gestão e o planejamento do Sistema Único de Saúde (SUS). Neste estudo, foi escolhida a Teoria Institucional como lente teórica para analisar a incorporação de SIS na saúde pública brasileira, uma vez que pode existir uma influência direta do ambiente organizacional. Esta perspectiva possibilita encontrar explicações à influência dos fatores ambientais, sejam eles de cunho coercitivo, normativo ou mimético, no momento da decisão do uso de tecnologias em governos. O objetivo do artigo é verificar a influência desses fatores institucionais na utilização dos SIS pelas Secretarias Estaduais de Saúde (SES). Para atendimento do objetivo da pesquisa, optou-se por uma abordagem qualitativa, com estudos de casos múltiplos nas SES dos estados do Paraná e Rio Grande do Sul, tendo como unidade de análise o Sistema de Informações de Mortalidade (SIM) e o Sistema de Informações Hospitalares Descentralizado (SIHD). Com base no modelo conceitual proposto, foram apresentados diferentes fatores institucionais que influenciam na utilização dos referidos SIS, na forma de pressões coercitivas, miméticas e normativas.

Palavras-chave: sistemas de informação em saúde (SIS); sistema único de saúde (SUS); teoria institucional.

\begin{abstract}
Health Information Systems (SIS) are technological artifacts allowing public managers in three government spheres to obtain essential information for the management and planning of the Brazilian Unified Health System (SUS - the public health system). Institutional Theory was chosen as the theoretical framework to analyze the incorporation of SIS in Brazilian public health, since there may be a direct influence from the organizational environment. This perspective makes it possible to find explanations for environmental factor influence, be they coercive, normative or mimetic, when deciding on the use of technology in government. The aim of this paper is to investigate the influence these institutional factors have on SIS use by State Health Departments (SES). In order to achieve our objective, we chose a qualitative approach, with multiple SES case studies in the states of Paraná and Rio Grande do Sul. The Mortality Information System (SIM) and the Decentralized Hospital Information System (SIHD) were considered the units of analysis for this study. Based on the proposed conceptual model, institutional factors that influence the use of these SIS, in the form of coercive, mimetic and normative pressures, are presented and analyzed.
\end{abstract}

Key words: health information systems (SIS); unified health system (SUS); institutional theory. 


\section{Introdução}

O desenvolvimento das Tecnologias de Informação e Comunicação (TIC) no ambiente governamental possibilita condições para o fornecimento mais efetivo de serviços públicos, dessa maneira, possibilitando a melhoria na gestão pública e o aprimoramento da criação de políticas públicas (Laia, Cunha, Nogueira, \& Mazzon, 2011). Diniz, Barbosa, Junqueira e Prado (2009) afirmam que entre os fatores que determinaram o uso extensivo das TIC pelos governos, para melhoria de seus processos internos e prestação de serviços, estão: a mudança do processo informacional centrado no papel para mídias eletrônicas; o uso dessas tecnologias de forma generalizada pela sociedade; o progresso da internet; e os fatores relacionados à Reforma do Estado, à modernização da gestão e à busca pela eficiência.

Seguindo esta tendência, a área da saúde pública brasileira, desde a década de 1970, vem desenvolvendo Sistemas de Informação em Saúde (SIS) com o objetivo de informatizar seus dados e, além disso, ter informações confiáveis para apoiar os processos de gestão dos serviços públicos em saúde. Os SIS são artefatos tecnológicos que respaldam gestores das três esferas governamentais a obterem informações imprescindíveis para apoiar a gestão e o planejamento do Sistema Único de Saúde (SUS).

Considerando que a incorporação de SIS na saúde pública brasileira pode ocorrer por influências diretas do ambiente organizacional, optou-se por utilizar nesta pesquisa a Teoria Institucional como lente teórica. Esta perspectiva teórica possibilita encontrar explicações quanto à influência dos fatores ambientais, sejam eles de cunho coercitivo, normativo ou mimético, no momento da decisão do uso das TIC em governos. A perspectiva institucional entende que as organizações inseridas em um mesmo campo organizacional sofrerão pressões que as tornarão mais homogêneas, para obterem legitimidade. Meyer e Rowan (1977) afirmam que as organizações sofrem transformações simbólicas e cerimoniais, que acarretam mitos institucionalizados no campo organizacional, ao invés dessas mudanças darem-se de forma racional, por meio de cálculos utilitaristas de custo e benefício.

Segundo Machado-da-Silva, Fonseca e Crubellate (2010), a teoria institucional tem sido adotada por pesquisadores do exterior e do Brasil, especialmente nas últimas décadas, pois a mesma tem sido bastante benéfica no domínio dos estudos organizacionais. Os autores defendem a abordagem recursiva do processo de institucionalização em que elementos como estrutura, agência e interpretação são tratados como noções fundamentais da institucionalização como processo recorrente condicionado por certo grau de estabilização dos sistemas sociais. Assim, os atores sociais, mediante sua capacidade de agência, participam no processo de construção social da realidade através de condicionantes estruturais, por meio do compartilhamento intersubjetivo de interpretações (Machado-da-Silva, Fonseca, \& Crubellate, 2010).

Esta pesquisa optou por usar a perspectiva institucional como lente teórica por entender que a utilização das TIC na esfera pública ocorre por influências contextuais peculiares ao seu campo organizacional, que vão além da adoção de práticas mais eficientes. Segundo Tolbert e Zulcker (1999), quando uma prática está institucionalizada no seu campo organizacional, ou seja, é legítima e reconhecida, sua estrutura foi propagada a todos os atores pertencentes ao campo e a sua estrutura está perpetuada por um considerável período de tempo.

O objetivo do artigo é verificar a influência dos fatores institucionais na utilização dos SIS pelas Secretarias Estaduais de Saúde (SES). Para atendimento do objetivo da pesquisa, optou-se por uma abordagem qualitativa de pesquisa, com estudos de casos múltiplos nas SES dos estados do Paraná (PR) e Rio Grande do Sul (RS), tendo como unidade de análise o Sistema de Informações de Mortalidade (SIM) e o Sistema de Informações Hospitalares Descentralizado (SIHD).

Após esta introdução, são apresentados aspectos relevantes do referencial teórico sobre SIS, a questão do campo organizacional e os processos isomórficos a partir da vertente sociológica da Teoria Institucional. Posteriormente, são detalhados os aspectos metodológicos utilizados para o 
desenvolvimento deste estudo. Na sequência, a análise e a discussão dos resultados são realizadas. Em seguida, os resultados são discutidos, sugerindo um modelo e proposições. Por fim, nas considerações finais, são discutidas algumas limitações do estudo e apresentadas sugestões de pesquisas futuras.

\section{Sistemas de Informação em Saúde}

Para Haux (2006), os SIS são todo e qualquer sistema que processa os dados, transformando-os em informação ou conhecimento na área da saúde, com a finalidade de colaborar para melhorar a qualidade da saúde da população. Os SIS distinguem-se dos Sistemas de Informações Hospitalares, pois, segundo o autor, trata-se apenas de uma unidade de um dos SIS por gerenciar apenas as informações em âmbito hospitalar. De forma mais ampla, a Organização Mundial de Saúde (OMS) define SIS como um SI que engloba a coleta, o processamento, a comunicação e o uso de informações essenciais para aprimorar a efetividade dos serviços de saúde (World Health Organization, 2004).

Por se tratar de uma área de estudo relativamente nova e em fase de formação, Mettler e Raptis (2012) propuseram uma estrutura de análise que lhes possibilitasse compreender o que constitui o campo de SIS. Ao construírem o que eles denominaram de uma grande fotografia coesa, identificaram três grandes temas e 18 subtemas como sendo um ponto de partida para uma agenda de pesquisa de SIS.

$\mathrm{Na}$ área da saúde, há uma preocupação latente por profissionais da área, governantes, entre outros atores, por equilibrarem os gastos e, ao mesmo tempo, impulsionarem a qualidade e o acesso aos serviços. Nesse contexto, o uso de SI configura-se em uma ferramenta com grande potencial para aprimorar questões relativas ao "custo-eficácia, qualidade e acessibilidade aos cuidados em saúde" (Chiasson \& Davidson, 2004, p. 156). Na mesma linha de raciocínio, Johnson (2011) afirma que os SIS podem oferecer ganhos econômicos, pois, através do gerenciamento adequado de dados, pode-se ter ganhos de eficiência na alocação dos recursos, visto que será possível, por meio dos dados, identificar possíveis gargalos na prestação e administração de cuidados na área da saúde.

Ronchi e Senne (2014) argumentam que o potencial do emprego de TIC na área da saúde promove uma melhoria na oferta dos serviços prestados na área. O registro dos dados neste campo tem sido valorizado cada vez mais, visto que se caracteriza como uma importante ferramenta para melhoria do atendimento. Outro avanço oriundo desses registros é a melhoria da eficácia do atendimento, o que permite acesso rápido de informações médicas dos pacientes.

Assim, é crescente o investimento financeiro para desenvolver novas TIC em saúde, mas uma parcela considerável é descartada em sistemas não implantados ou ineficazes (Heeks, 2006). Ainda abordando questões como a implementação das TIC em saúde, Spil, LeRouge, Trimmer e Wiggins (2009) afirmam que é visível que as TIC na área da saúde são agentes facilitadores das mudanças organizacionais tanto em âmbito local quanto em âmbito nacional. Contudo, devido à inúmera variedade ofertada no mercado, a adoção dessa tecnologia é complexa.

As TIC nesse ambiente têm sido utilizadas nas mais diversas esferas no que tange aos ambientes em saúde, como: Prontuário Eletrônico (Bansler \& Havn, 2010), Sistema de Informação em Hospitais (Haux, 2006), Telemedicina (Jones et al., 2012), entre outras. Essas tecnologias possuem um enorme potencial para propulsionar melhorias que envolvem ganhos de qualidade e acessibilidade no sentido dos cuidados em saúde e a melhoria considerável do custo-benefício (Souza, Cunha, \& Dornelas, 2011).

No contexto brasileiro, destaca-se também o uso das TIC, na esfera pública de saúde, promovendo uma gestão mais qualificada das informações neste âmbito, com o intuito de apoiar os gestores no momento de planejar e desenvolver políticas públicas. Contudo, como demonstram os resultados alcançados por Vidor, Fisher e Bordin (2011, p. 29), "os SIS mais frequentemente alimentados e analisados relacionavam-se com controle orçamentário ou repasses financeiros do nível central". 


\section{Campo Organizacional e os Processos Isomórficos}

O neoinstitucionalismo, considerado uma nova roupagem da Teoria Institucional, cresceu e difundiu-se a partir da década de 1970 e tem realizado significativas contribuições quanto ao entendimento da dinâmica das organizações contemporâneas (Frunkim \& Galaskiewicz, 2004). Os estudos de cunho institucionalista vão ao encontro da visão racionalista do comportamento organizacional, pois é reconhecido, neste ambiente, que as organizações estão inseridas em um amplo contexto cultural e político que influencia as suas ações.

O marco dessa teoria se dá por meio de processos de socialização, aprendizado ou aderência a convenções existentes no contexto em que se está inserido (Laia et al., 2011). Nessa perspectiva, a todo o momento, as pessoas realizam escolhas, porém não se dão conta de que ocorrem a partir da busca por referências de outros atores em situações similares e também através de modelos de referências, a partir de padrões existentes no campo organizacional (DiMaggio \& Powell, 1991).

Desse modo, as organizações são vistas como um aglomerado de normas, padrões e valores mergulhados na realidade cultural da instituição (Laia et al., 2011). Complementando este argumento, Mignerat e Rivard (2009) afirmam que, tanto organizações quanto seus atores organizacionais, agem com o intuito de legitimar, no ambiente em que estão inseridos, assim, garantindo a sua perenidade nesse ambiente.

O tema institucionalização está fortemente ligado à busca da legitimidade pelas organizações (Deephouse \& Suchman, 2008). Na definição de Suchman (1995, p. 574), "legitimidade é a percepção ou pressuposição generalizada de que as ações de uma entidade são desejáveis ou apropriadas dentro de algum sistema socialmente construído de normas, valores, crenças e definições". Esse processo de institucionalização aportado pela busca da legitimidade se dá pelo fato de que as organizações necessitam, em um campo organizacional, desenvolver uma credibilidade, bem como uma aceitação, frente a seus stakeholders (Scott, 2008). A legitimidade é alcançada pelas organizações, pois elas buscam, implícita ou explicitamente, uma homogeneidade no campo organizacional, através dos processos isomórficos.

Antes de expor as definições de campo organizacional e processos isomórficos, é necessário abordar dois conceitos importantes quando se utiliza a lente institucional para a análise de um fenômeno: organizações e instituições. Para Selznick (1957), o conceito de organização possui uma conotação técnica, em que é concebida como um meio para atingir objetivos. Por sua vez, o conceito de instituição possui um significado mais amplo, visto que ela é o resultado de processos de interação e adaptação, sendo o produto naturalmente constituído por meio das necessidades e das pressões sociais, desse modo, caracterizando-se por um sistema social.

Machado-da-Silva et al. (2010) consideram que a institucionalização é melhor analisada sob uma ótica multiparadigmática, em que os atores desenvolvem ações nas organizações por meio da interpretação de diferentes fatores, que devem ser legitimadas através do compartilhamento e da socialização. Para os autores, a homogeneização deriva da aceitação de ações que são interpretadas como legítimas e, consequentemente, são reproduzidas pelos agentes na prática social, consolidando-se como padrão institucionalizado. Legitimidade é o elemento que permite a manutenção ou a mudança nas organizações, em que "questionamentos a respeito da adequação de práticas, normas e procedimentos em razão de pressões internas e externas podem impossibilitar a reprodução dos padrões institucionalizados, acarretando a perda da sua legitimidade" (Machado-da-Silva et al., 2010, p. 98).

Desenvolvimentos da teoria institucional nos anos de 1970 e 1980 chamaram a atenção para a significação de formas organizacionais e campos organizacionais, sendo as organizações identificadas como tipos distintos de formas sociais (Scott, 2008). O conceito de campo organizacional é definido como um local em que as organizações constituem uma área reconhecida no ambiente organizacional, que possui "fornecedores-chave, consumidores de recursos ou produtos, agências regulatórias e outras organizações que produzam serviços e produtos similares" (DiMaggio \& Powel, 1983, p. 148). A estrutura de um campo não pode ser determinada de forma prévia, mas é necessário que essa seja constituída a partir de 
investigações empíricas, pois passam a existir somente a partir do momento que podem ser definidos institucionalmente (DiMaggio \& Powel, 1983).

Estes autores afirmam que "uma vez que diferentes organizações, no mesmo ramo de negócios, estejam estruturadas em um campo concreto ..., forças poderosas emergem, levando-as a tornarem-se mais similares às outras" (DiMaggio \& Powel, 1983, p. 148) e, a partir disso, dentro de um campo, as organizações podem vir a modificar a sua conduta, de forma a alterar suas metas ou até mesmo desenvolver novas práticas de trabalho. Esse processo de tornar as organizações dentro de um campo mais similares é denominado isomorfismo.

Segundo Fonseca (2003), há dois tipos de isomorfismo: o competitivo e o institucional. O primeiro é oriundo de um processo competitivo e de pressões mercadológicas, e o segundo inclui a luta das organizações pela legitimidade em seu campo de atuação. Este trabalho detém-se na perspectiva institucional que busca a legitimação das organizações. DiMaggio e Powell (1983) identificaram três mecanismos que provocam mudanças isomórficas dentro do campo, são eles: o isomorfismo coercitivo, o isomorfismo mimético e o isomorfismo normativo. Adicionalmente, Scott (2008) define tais influências isomórficas por meio de três pilares: o pilar regulador, o pilar cultural-cognitivo e o pilar normativo. Ambas as abordagens, a de DiMaggio e de Powell (1983) e a de Scott (2008), serão detalhadas nas próximas subseções.

\section{O isomorfismo coercitivo e o pilar regulador}

O isomorfismo coercitivo pode ocorrer por pressões formais ou informais exercidas em uma organização por outras organizações das quais aquela dependa e por expectativas culturais oriundas da sociedade (DiMaggio \& Powel, 1983). Em certas ocasiões, as mudanças organizacionais podem vir por meio de sanções governamentais e de legislações que de forma coercitiva interferem no campo. A existência de aspectos legais em um campo organizacional provoca mudanças nas organizações, sejam elas de cunho estrutural ou comportamental. O resultado dessa normatização do campo organizacional acaba por tornar as organizações mais homogêneas "dentro de determinados domínios e cada vez mais organizadas em torno de rituais em conformidade com instituições maiores" (DiMaggio \& Powel, 1983, p. 150).

De forma semelhante, os sistemas reguladores "envolvem a capacidade de estabelecer regras, inspecionar outros em conformidade com elas e, se necessário, manipular sanções - em uma tentativa para influenciar um comportamento futuro" (Scott, 2008, p. 52). São processos ligados a leis, normas ou sanções que acarretam pressões no campo organizacional para que ocorra uma adequação frente a essa imposição.

De acordo com Scott (2008), os estudiosos do campo sustentam que as instituições tanto restringem quanto regularizam o comportamento em seu ambiente. Outra ponderação realizada pelo autor é que apesar de o pilar regulador transparecer ser ligado a práticas de repressão e constrangimento, não apenas em leis está respaldado o seu poder, mas também em regulações que habilitam os atores sociais e suas ações, como licenças, benefícios para o mesmo tipo de atores e poderes especiais que podem ser delegados a um grupo.

Segundo Machado-da-Silva, Guarido, Nascimento e Oliveira (2003, p. 182) "a vertente que se enquadra no pilar regulativo baseia-se em pressupostos realistas sociais". Os autores argumentam que os atores sociais conduzem seus interesses a partir de um olhar racional utilitarista, em que buscam o custobenefício. Com o intuito de evitar divergências, regras e leis são estabelecidas e sanções são impostas. Dessa forma, normas, leis e sanções constituem a base de legitimação institucional.

Infere-se, portanto, que tanto o isomorfismo coercitivo de DiMaggio e Powell (1983) como o pilar regulador de Scott (2008) remetem a pressões às quais as organizações são submetidas de forma impositiva, pois, no momento em que são estabelecidas normas, leis e sanções no campo organizacional, os atores pertencentes a esse campo são obrigados a incorporar essa normativa em busca de sua legitimidade e padronizações no ambiente em que convivem. 


\section{O isomorfismo mimético e o pilar cultural-cognitivo}

O isomorfismo mimético é proveniente da incerteza que, dentro de um campo organizacional, estimula o processo de imitação. Nesse sentido, "as organizações tendem a tomar como modelo em seu campo outras organizações, que elas percebem ser mais legítimas ou bem sucedidas" (DiMaggio \& Powel, 1983, p. 150). Segundo DiMaggio e Powel (1983), quando, no campo organizacional, estabelecese um processo de incerteza, devido a metas ambíguas, as organizações tendem a tomar outras intuições como modelo. Para os autores, o fato de tomar outras organizações como modelo é uma resposta à incerteza vinda do campo. Por sua vez, a organização que está sendo imitada, muitas vezes, não sabe que está sendo tida como modelo e, até mesmo, não gostaria de ser imitada (DiMaggio \& Powel, 1983).

De forma similar, o sistema cultural-cognitivo possui relação com convicções comuns, lógicas compartilhadas e é apoiado pela cultura. As interações existentes em um campo organizacional acabam por reconhecer que "processos interpretativos 'internos' são moldados por estruturas culturais "externas"” (Scott, 2008, p. 57). Esses processos interpretativos ou, conforme Ranson, Hinings e Greenwood (1980) denominam, de esquemas interpretativos definem a maneira como indivíduos que compõem as organizações percebem e compreendem as informações vindas do contexto externo ou do seu próprio campo organizacional.

O pilar cultural-cognitivo está ligado a questões miméticas, em que, frente às incertezas, as organizações irão captar, no ambiente, padrões de conduta que, de certa forma, são tomados como a melhor maneira de operar em seu campo (Scott, 2008). Assim, define-se que a concepção de instituições, que são regidas pelo pilar cultural-cognitivo, dá ênfase ao papel central dos padrões socialmente construídos (Scott, 2008).

\section{O isomorfismo normativo e o pilar normativo}

O isomorfismo normativo, segundo DiMaggio e Powel (1983), é derivado primordialmente do processo de profissionalização. Esse processo ocorre pelo esforço em conjunto de pessoas de uma mesma profissão em prol de uma definição quanto às condições e formas de trabalho, com o intuito de ter o controle do produto final dessa categoria.

De acordo com DiMaggio e Powell (1983, p. 150), dois aspectos "da formalização são fontes importantes de isomorfismo". Em primeiro lugar, o aspecto educacional, da legitimidade de uma base cognitiva produzida por especialistas universitários. E, em segundo lugar, a constituição de redes profissionais, nas quais novos modelos ou práticas são rapidamente difundidos, pois perpassam as fronteiras organizacionais. Nesse contexto, universidades e associações profissionais são ambientes que desenvolvem regras de comportamento organizacional e também profissional.

De maneira semelhante, os sistemas normativos determinam como as coisas devem ser feitas. Nesse tipo de sistema são definidas metas e objetivos, bem como a forma como devem ser executados tais processos para busca dos objetivos pré-definidos (Scott, 2008). O pilar normativo está condicionado a questões de certificação e credenciamento, visto que se podem vincular a esse sentido categorias profissionais, em que se espera dos atores sociais envolvidos atitudes e performances semelhantes de acordo com prescrições de como eles devem se portar (Scott, 2008).

\section{Metodologia}

O presente estudo optou por apropriar-se do enfoque qualitativo para análise dos casos. Esse tipo de abordagem tem como característica examinar de maneira detalhada a forma como os indivíduos constroem o mundo em seu contexto, por meio de suas vivências cotidianas, e, com isso, conseguir entender e descrever o fenômeno social a partir da perspectiva interna. $O$ pesquisador que opta por esse tipo de abordagem possui interesse em estabelecer contato com as experiências, documentos e interações 
em seu ambiente natural, sem interferir na realidade observada (Gibbs, 2009). A respeito da estratégia escolhida para realização da pesquisa, foi definida a utilização de estudo de casos comparativos. Para Godoy (1995), o estudo de caso deve focar em uma situação ou evento particular, em que a importância do estudo é oriunda daquilo que o caso revela sobre o objetivo investigado.

Como forma de delimitação do escopo da pesquisa, foram selecionados os sistemas SIM e SIHD utilizados nos estados do Paraná e do Rio Grande do Sul. O critério adotado para a seleção dos estados foi o fato de ambos se localizarem na região Sul do país. Outro fator que facilitou a escolha dos municípios foi a indicação de contatos nesses locais para articular as entrevistas. Os ambientes, foco do estudo, foram as Secretarias Estaduais de Saúde dos referidos estados, uma vez que esses sistemas são utilizados pelas secretarias e por departamentos específicos. No Brasil, o órgão responsável pelo fomento de SIS e gestão dos dados na área da saúde é o Departamento de Informática do SUS (DATASUS).

Os critérios para a escolha dos SIS investigados ocorreram com base nos seguintes fatores motivadores: (a) SIS ofertado pelo DATASUS às Secretarias de Saúde; (b) SIS ser um sistema de base nacional do DATASUS, caracterizando-se como Governo Eletrônico do tipo e-Administração; (c) SIS significativo para gestão das ações e serviços em saúde. Estabeleceram-se, ainda, duas categorias de sistemas que envolvessem aspectos importantes da gestão do SUS: (a) aspectos epidemiológicos e (b) aspectos financeiros e de remuneração.

Assim, a partir da categorização dos SIS para efetuar a escolha e com base nos fatores motivadores citados, os sistemas SIM e SIHD foram escolhidos para este estudo. O SIM é um sistema que possui a finalidade de obtenção de dados regulares sobre mortalidade no Brasil. Já o SIHD contém informações que viabilizam efetuar o pagamento dos serviços hospitalares prestados pelo SUS, pela captação de dados das Autorizações de Internação Hospitalar.

A Unidade de Análise deste estudo foram os Sistemas de Informação em Saúde (SIM e SIHD) ofertados pelo DATASUS, caracterizados como sistemas de apoio à gestão e ao planejamento governo a governo.

\section{Objeto de estudo: sistemas do DATASUS}

O DATASUS foi criado em abril de 1991, com base no Decreto $\mathrm{n}^{\circ} 100$. Este decreto regulamentou a transferência de funcionários que compuseram o quadro de servidores da Fundação Nacional de Saúde (FUNASA) - oriundos da Diretoria de Sistemas de Saúde da Empresa de Tecnologia e Informações da Previdência Social (DATAPREV), da Fundação Serviços de Saúde Pública (Fundação SESP) e da Superintendência de Campanhas de Saúde (SUCAM). Dessa forma, foi retirada da DATAPREV a função específica de controle e processamento das contas referentes ao setor Saúde, que passaram a ser de responsabilidade do Ministério da Saúde (MS), por delegação atribuída à FUNASA, por meio do seu Departamento de Informática.

Somente em 1998, o DATASUS passou a vincular-se ao Ministério da Saúde, devido às necessidades desse órgão em ter um departamento com tais características. Nesse período, o MS passou por mudanças em sua estrutura e missão, passando a ter o entendimento da importância da informação tanto para os processos de gestão quanto para a formulação de políticas públicas.

O DATASUS possui como grande atribuição gerenciar as informações da área da saúde no Brasil. Para isso, ele disponibiliza para secretarias municipais e estaduais de saúde SI que fornecem informações imprescindíveis para o desenvolvimento de uma boa gestão em saúde de acordo com as peculiaridades de cada região. Para que estes sistemas sejam ferramentas cruciais para a gestão da saúde, é preciso que sejam supridos de dados qualificados, pois, a partir das informações geradas nestes sistemas, dar-se-á o planejamento da oferta de serviços de saúde, juntamente com a alocação dos recursos disponíveis. 
De acordo com o site do DATASUS, atualmente, estão disponíveis para uso da esfera pública 39 sistemas distintos, estes agrupados em nove categorias. São elas: (a) ambulatoriais; (b) epidemiológicos; (c) financeiros; (d) hospitalares; (e) estruturantes; (f) eventos vitais; (g) tabulação; (h) sociais; (i) regulação. Em função do foco deste estudo, a seguir, é detalhado o histórico do SIM e do SIHD, sistemas de base nacional desenvolvidos e mantidos pelo DATASUS (http://www2.datasus.gov.br/DATASUS/index.php?area=06, recuperado em 15, agosto, 2014).

\section{O histórico do sistema de informação sobre mortalidade}

Há indícios, no Brasil, da preocupação com registros de óbito desde 1914, quando foi sancionada a Lei $\mathrm{n}^{\circ} 1870$ que criou a Diretoria Geral de Estatística, que possuía como competência a gestão de dados sobre nascimentos, casamentos e óbitos (Jorge, Laurenti, \& Gotlieb, 2009). Em 1973, a Lei ${ }^{\circ}$ 6.015 sanciona que todo óbito ocorrido no país deveria obrigatoriamente ser registrado em cartório.

Fatos que ocorreram nas décadas de 1960 e 1970 ajudaram a impulsionar a conscientização das autoridades competentes sobre a importância da criação de um sistema de base Nacional sobre informações de mortalidade, a saber (Jorge et al., 2009):

1. atuação da Fundação Serviço Especial de Saúde Pública (Sesp) em trabalhos sobre estatísticas vitais no norte e nordeste do país;

2. atuação da Faculdade de Saúde Pública da Universidade de São Paulo (USP) em ministrar cursos de Classificação Internacional de Doenças (CID-10) datados desde a década de 1960; participação em estudos coordenados pela Organização Pan-americana de Saúde (OPAS) e Organização Mundial de Saúde (OMS) sobre mortalidade em adultos e mortalidade infantil;

3. criação de SI estaduais sobre mortalidade e a inexistência de informações de óbitos no Brasil nos Anuários Internacionais da OPS, OMS e Nações Unidas.

Vistos os pressupostos favoráveis à construção do sistema, em 1975, O Ministério da Saúde percebendo a importância de um SI dessa natureza, preconizou a implantação de um desses SIS, em que existiria um subsistema de mortalidade com dados abrangentes e confiáveis. A partir disso, no mesmo ano, foi criada uma equipe de trabalho para apoiar a concepção do sistema. Essa equipe verificou que em todo país havia diferentes tipos de atestados de óbito (com informações distintas) e também diferentes fluxos dessas informações até chegar ao órgão competente (Jorge et al., 2009).

Tendo em vista tais constatações, o grupo de trabalho recomendou a adoção de um modelo único de Declaração de Óbito (DO) em todo o Brasil, o qual deveria seguir um fluxo padronizado. Outra sugestão foi de que isso deveria ser feito em âmbito central, pois na época tinha-se a ideia de que as ações deveriam ser sempre centralizadas (Jorge et al.,2009).

Com o intuito de divulgar essas decisões e obter adesões em âmbito regional, nesse mesmo ano, foi realizada a primeira reunião nacional sobre SIS. No evento, foi apresentada a situação atual das informações em saúde e as perspectivas para o futuro, bem como o novo modelo de DO para o subsistema de informação de mortalidade (Brasil, 1984-1998 como citado em Jorge et al., 2009). Nessa convenção, aprovou-se o modelo único de DO, os fluxos que deveriam ser seguidos e os dados a serem sistematizados, "ficando claro que, com base na experiência já existente no estado do Rio Grande do Sul", cada unidade da federação poderia ampliar o número de variáveis a serem tabuladas (Jorge et al., 2009, p. 73).

Como consequência, iniciou-se o processo de implementação do SIM em todo o país. Para tanto, foram desenvolvidos materiais didáticos para apoio à utilização do sistema e codificação da causa da morte. Também, foi realizado um forte e amplo treinamento que, além de trabalhar na sensibilização das autoridades envolvidas, incluía o processo de convencimento de médicos no preenchimento correto da DO, bem como a capacitação dos responsáveis pelo processamento e análise dos dados (Jorge et al., 2009, p. 73). 
Em virtude das diversas mudanças estruturais ocorridas no Ministério da Saúde ao longo dos anos, o SIM esteve sob responsabilidade de diferentes setores desde sua a concepção. Desde 2003, o sistema está sendo gerenciado pela Secretaria de Vigilância em Saúde (SVS). Contudo, somente no ano de 2000, por meio da Portaria n. 474 - FUNASA (posteriormente substituída pela Portaria n. 20, de 2003), foi estabelecido o primeiro marco legal do SIM. Nessa legislação, além do estabelecimento da obrigatoriedade em enviar informações sobre mortalidade, foram regulamentadas questões referentes à coleta de dados, ao fluxo das informações e à periodicidade do seu envio.

\section{O histórico do sistema de informações hospitalares}

Na década de 1970, os pagamentos efetuados aos hospitais privados contratados pelo MS eram realizados por meio do Sistema Nacional de Controle e Pagamentos de Contas Hospitalares (SNCPCH), que utilizava a Guia de Internação Hospitalar (GIH) como instrumento de entrada dos dados no sistema. Assim, a principal característica do SNCPCH era a de realizar o pagamento das despesas de internação dos hospitais contratados e pagos via recursos públicos.

Em 1981, o sistema foi aprimorado e passou a ser denominado de Sistema de Assistência MédicoHospitalar da Previdência Social (SAMHPS). Inicialmente, o SAMHPS foi utilizado de forma experimental na cidade de Curitiba e, em seguida, disseminou-se pelo estado do Paraná. Em 1983, o sistema já era utilizado em todo o país para o pagamento dos hospitais contratados. Segundo Pepe (2009, p. 65), o valor do repasse de verbas a essas instituições era baseado "em um sistema de remuneração fixa por procedimento, considerando valores médios, pré-fixados, de procedimentos realizados na rede de assistência hospitalar". Nesse momento, foi criado o formulário, que ainda é utilizado, denomina-se Autorização de Internação Hospitalar (AIH).

A AIH é o instrumento pagador das internações hospitalares que é distribuído aos estados numa proporção de nove por cento da população residente, podendo ser estabelecido um teto financeiro para o pagamento das internações. Os estados são responsáveis pela distribuição dos recursos aos municípios dessas AIHs, de acordo com as pactuações realizadas entre os gestores (Pepe, 2009).

Imediatamente após a implantação do SUS, em 1990, a AIH passou a ser chamada de Sistema de Informações Hospitalares (SIH). Contudo, somente após o ano de 1991, diversas alterações importantes foram introduzidas no sistema, destacando-se aquelas ligadas ao processo de descentralização para secretarias de saúde e para os prestadores, no caso os hospitais (Carvalho, 2009).

Em 2004, foi iniciado o processo de descentralização do SIH (Portaria n. 821, 2004). Justificava-se a necessidade de descentralizar o processamento dos dados do SIH para gestores estaduais, do Distrito Federal e dos municípios em gestão plena, objetivando acompanhar o processo de descentralização da gestão da saúde pública brasileira, que iniciou na década de 1990.

Logo em seguida, foi criado um novo sistema descentralizado, o Sistema de Informações Hospitalares Descentralizado (SIHD), o qual teve um ano para ser implementado pelos entes federados e prestadores de serviço (Portaria n. 510, 2005). O SIHD possui dados do Cadastro Nacional de Estabelecimento de Saúde (CNES) e utiliza o CID-10 (Código Internacional de Doenças) para classificação das morbidades das internações hospitalares, informação importante para constatação dos motivos de internação dos pacientes. Em 2003, o CNES conseguiu unificar e substituir completamente diversos cadastros de estabelecimentos de saúde existentes utilizados pelos SIS de base nacional.

O CNES também disponibiliza informações sobre a área física, recursos humanos, equipamentos, serviços de apoio diagnóstico e terapêutico e serviços ambulatoriais e hospitalares das unidades de saúde. Abrange a totalidade dos hospitais existentes no país, assim como a totalidade dos estabelecimentos ambulatoriais vinculados ao SUS e, ainda, grande parte dos estabelecimentos ambulatoriais não vinculados ao SUS (Pepe, 2009). 


\section{Coleta e análise de dados}

A coleta de dados foi realizada com base em entrevistas em profundidade, individuais e em grupo, utilizando roteiro semiestruturado. Também, foram utilizados dados provenientes da análise de documentos, apoiada no referencial teórico desenvolvido nas seções anteriores. A escolha dos indivíduos entrevistados foi realizada em consonância com o foco de investigação deste trabalho, visto que possui como problemática de pesquisa a utilização de SIS para apoio à gestão e ao planejamento do SUS. Por essa razão foram entrevistados indivíduos responsáveis pela utilização e gestão das informações nos SIS investigados nas Secretarias Estaduais de Saúde dos estados do RS e PR. Para tanto, priorizou-se a realização de entrevistas com pessoas que acompanharam a evolução da utilização desses SIS.

Uma vez que os sistemas investigados são alocados em departamentos distintos, foram realizados contatos com áreas diferentes dentro das SES. Além de representantes das SES, foram entrevistados também profissionais que trabalham no DATASUS no estado do PR e RS, assim como o Coordenador de Disseminação de Informações do DATASUS central. Foram realizadas 13 entrevistas entre 20 de outubro e 10 de dezembro de 2012. Cada um dos entrevistados será referido neste texto como E1, E2, $\mathrm{E} 3, \ldots, \mathrm{E} 13$.

Para executar a análise dos dados coletados, foi utilizada a técnica de análise de conteúdo. Bardin (2011) define a análise de conteúdo como um conjunto de instrumentos metodológicos, que se encontra em constante aperfeiçoamento e aplicável a discursos bastante diversificados. A análise de conteúdo foi organizada em três fases definidas como: pré-análise; exploração do material; e tratamento dos resultados obtidos e interpretação (Bardin, 2011). A dimensão definida para análise dos dados foi subdividida em: (a) influência de fatores institucionais na utilização do SIM e (b) influência de fatores institucionais na utilização do SIHD. Os fatores institucionais foram analisados por meio dos isomorfismos coercitivo, normativo e mimético.

$\mathrm{Na}$ fase de tratamento dos resultados, proposta Bardin (2011), os resultados brutos foram tratados para que fossem "significativos ('falantes') e válidos" (p. 131), sendo delimitado um modelo de resultados que condensou e colocou em relevo as informações obtidas na análise.

Para o tratamento dos resultados, foram realizadas inferências com base nas falas analisadas. Nas palavras de Bardin (2011, p. 131), "o analista, tendo à sua disposição resultados significativos e fiéis, pode então propor inferências e adiantar interpretações a propósito dos objetivos previstos - ou que digam respeito a outras descobertas inesperadas."

Assim, a partir de aspectos advindos da fundamentação teórica da pesquisa, foram descritos os resultados obtidos com entrevistas realizadas nas Secretarias de Saúde dos dois estados. As próximas seções apresentam o resultado da $3^{\mathrm{a}}$ fase da análise de dados, por meio da análise e discussão dos dados e apresentação dos resultados da pesquisa.

\section{Análise e Discussão dos Dados}

Com base nas dimensões definidas para análise dos dados, esta seção apresenta a discussão da influência dos fatores institucionais (por meio dos diferentes isomorfismos) na utilização dos SIS analisados.

\section{A influência dos fatores institucionais na utilização dos SIS}

A preocupação com a organização das informações em saúde ocorreu de forma desordenada até o início da década de 1970, quando inúmeras discussões a respeito da sistematização das informações em saúde emergiram. Em 1970, o Planejamento, Avaliação, Pesquisa e Programas Especiais (PAPPE), secretaria vinculada ao MS, recebeu a incumbência de promover a criação do setor de informática na saúde 
(Branco, 2006). Assim, em 1973, foi promovida uma reunião organizada com o PAPPE juntamente com OPAS e seis estados brasileiros. Nesta ocasião foram estudados e debatidos um conjunto de documentos elaborados pelo PAPPE e pela OPAS sobre os SIS no Brasil. Firmou-se, assim, que cada um dos seis estados iria assumir um projeto de informatização de uma área específica da saúde. Na ocasião, o estado do Rio Grande do Sul ficou responsável pelo desenvolvimento de um sistema com enfoque epidemiológico (Branco, 2006).

Com base nesta contextualização, foi possível verificar a influência de pressões externas para o fomento das iniciativas de informatização dos dados sobre a saúde da população. As pressões que ocorreram nesse processo podem ser determinadas como miméticas, coercitivas e normativas (Scott, 2008).

Neste estudo, as pressões miméticas podem ser consideradas aquelas oriundas do contexto internacional, em que organizações como a OMS e OPAS, que possuem como meta melhorar a situação de saúde da população mundial e das Américas, respectivamente, estavam sistematizando as informações em saúde em outros países. Na década de 1960, a OMS já publicava anuários sobre mortalidade, informações que até então não estavam informatizadas no país (Jorge et al., 2009). Dessa forma, devido à situação de incertezas frente à gestão das informações em saúde no país, que ainda se encontrava desordenada e era proveniente de intervenções pontuais (Ministério da Saúde, 2009), foi necessário adequar-se ao contexto internacional, pois sistematizar informações poderia promover melhores condições de saúde à população.

Como pressão coercitiva, também oriunda do contexto internacional, pode ser atribuído o fato do Brasil ser país-membro de ambas as organizações, OMS e OPAS, inclusive da ONU, desde 1945, e acaba por aderir às determinações e aos acordos propostos por essas organizações. Outra pressão coercitiva pode ser atribuída à assinatura de termo entre os seis estados participantes da reunião realizada pela OPAS e pelo PAPPE, em 1973. Esta reunião teve como objetivo discutir sobre SIS e firmar projetos de informatização de dados sobre saúde com os estados convidados, de acordo com documentos elaborados por estas instituições (Branco, 2006).

Nas seções a seguir, são expostos os fatores que levaram os sistemas analisados (SIM e SIHD) a serem desenvolvidos e incorporados por estados, municípios e prestadores. Também, são verificadas certas peculiaridades nesses sistemas impostas por questões contextuais e influências do meio ou, como prefere Scott (2008), influências do campo organizacional.

\section{A influência de fatores institucionais na utilização do SIM}

O SIM foi o primeiro SIS de base nacional a ser desenvolvido no Brasil. A sua concepção deu-se em razão da necessidade de padronizar e informatizar dados sobre mortalidade no país. Contudo, antes da concepção desse sistema, o RS já havia desenvolvido um registro informatizado de dados sobre mortalidade com sucesso. Um dos entrevistados paranaenses (E12) lembra dessa experiência e relata: "o RS tinha o sistema antigo, processado em um mainframe. O RS foi um dos primeiros estados a registrar eletronicamente os atestados de óbito, antes do Brasil inclusive, e nós fomos buscar essa tecnologia deles e processamos algumas coisas nesse sistema do RS".

Assim, constata-se que o RS foi pioneiro ao realizar o registro eletrônico dos atestados de óbito, antes mesmo do Brasil instituir o SIM. Essa experiência foi tão positiva que representantes do estado do PR vieram ao RS para conhecer a tecnologia e até utilizaram-na para informatizar seus dados. Esse tipo de modelo de sucesso caracteriza-se como uma influência mimética no campo organizacional, visto que, frente às incertezas a respeito da padronização desses dados, despontou essa experiência positiva no ambiente nacional.

Ainda na década de 1970, ocorreu a primeira reunião sobre Sistemas de Informação em Saúde, em que um dos itens constantes na pauta era desenvolvimento de um Sistema de Informação sobre Mortalidade. Nessa reunião se institucionalizou o modelo único de declaração de óbito, que foi 
estabelecido com base na experiência do RS (Jorge et al., 2009). Mais uma vez, vê-se a ação de pressões miméticas do caso bem-sucedido do RS influenciando a concepção do SIM.

Criado o sistema, iniciou-se então o processo de implementação do SIM em todo o país. Na ocasião, foram desenvolvidos materiais de apoio, como manuais, e também treinamentos intensivos sobre a utilização do sistema e classificação da causa de morte (Jorge et al., 2009).

Para utilizar o sistema é necessário que o profissional seja treinado, tanto para operá-lo como para aprender a codificar a causa morte. O depoimento do E1 revela pontos sobre essa busca pela profissionalização:

“Antigamente tinha muito Cargo de Confiança (CC) envolvido, daí nós perdíamos muito treinamento. Nós treinávamos uma pessoa daí, quando assumia, o outro prefeito, ele tirava aquele $\mathrm{CC}$ e nós tínhamos que treinar de novo outra pessoa. Então, hoje em dia, a gente pede um funcionário de carreira para ser treinado, um servidor concursado".

Devido a essa descontinuidade constante de pessoas que não possuem vínculo empregatício, ao contrário do servidor concursado, e que a cada troca de governo há mudança no efetivo desse segmento, institucionalizou-se que o treinamento no RS deve ser dado a servidores com vínculo ao órgão onde trabalha. Os treinamentos caracterizam um processo de profissionalização das pessoas que utilizam esses sistemas, pois, além de deterem conhecimento sobre a manipulação da ferramenta, devem ter um curso específico para aprender sobre codificação da causa morte, fator de extrema importância para qualificar o dado inserido no sistema.

Assim, verifica-se nesse processo a influência de pressões normativas, pois é visível o esforço das pessoas envolvidas na definição das condições e formas de trabalho, com o objetivo de ter o controle do produto final, no caso, as informações em saúde.

Outro fato importante que confirma a grande disseminação do SIM pode ser constatado ao acessar o site do DATASUS. Nas informações de saúde da tabulação via Internet/Intranet (TABNET), são apresentados dados sobre mortalidade desde o ano de 1979, de todos os estados brasileiros, o que demonstra que essas informações são compiladas desde o final da década de 1970 (http://www2.datasus.gov.br/DATASUS/index.php?area=0205, recuperado em 15, agosto, 2014).

Importante também destacar que o SIM utiliza o Código Internacional de Doenças (CID-10) para padronizar a codificação das causas de morte. Esse sistema de codificação foi concebido pela Organização Mundial de Saúde para classificação de doenças e outros registros de saúde, incluindo certidões de óbito (World Health Organization, 2012). O relato do E1 evidencia esse fato. "Existe o Código Internacional de Doenças, que é a CID-10 atual, essa classificação internacional de doenças é usada no mundo inteiro, se I21 aqui é infarto agudo do miocárdio, na China também vai ser".

A adoção dessa codificação reconhecida internacionalmente pode ser determinada como uma pressão normativa, visto que a CID é uma classificação amplamente reconhecida internacionalmente e desenvolvida e mantida pela OMS, organização a qual o Brasil é membro. Apesar de não ser uma exigência da OMS, a conformidade com padrões estabelecidos pode ser vista como uma influência coercitiva do meio em se tratando de uma prática institucionalizada e reconhecida mundialmente. A utilização desse código permite que as informações sobre mortalidade no Brasil sejam comparadas a outras de qualquer país que adote o mesmo código, havendo uma homogeneização das informações de mortalidade em âmbito internacional.

Conquanto o SIM seja um sistema utilizado pelos estados brasileiros há mais de três décadas, somente em agosto de 2000, instituiu-se o marco legal quanto à obrigatoriedade do envio de informações sobre mortalidade. A Portaria n. 20, de 2003, estipula, em seu Art. 24, que:

A falta de alimentação de dados no Sistema de Informações sobre Mortalidade - SIM, ... por mais de 60 dias, ensejará a suspensão das transferências dos recursos do Piso de Atenção Básica PAB e o cancelamento da Certificação para Gestão das Ações de Epidemiologia e Controle de Doenças, bem 
como a consequente suspensão do repasse dos recursos do Teto Financeiro de Epidemiologia e Controle de Doenças - TFECD. (p. 71).

Não há dúvida de que esta portaria representa uma pressão coercitiva para a remessa periódica dos dados sobre mortalidade. O não cumprimento dessa portaria acarreta corte da transferência de recursos do Piso de Atenção Básica (PAB), o cancelamento da Certificação da Gestão das Ações Epidemiológicas e controle de doenças, além da interrupção do repasse de recursos do Teto Financeiro de Epidemiologia e Controle de Doenças. Assim, essa portaria determinou a remessa compulsória dos dados e a não observância dessa determinação ocasiona o corte de recursos ao ente federado que a descumpriu.

\section{A influência de fatores institucionais na utilização do SIHD}

Apesar de ter sido criado, na década de 1970, um sistema com o objetivo de processar o faturamento dos hospitais contratados e pagos por recursos estatais, o SIHD só foi desenvolvido e disseminado em 2006. Antes disso, várias formulações desse sistema existiram com nomes distintos, como o Sistema Nacional de Controle e Pagamentos de Contas Hospitalares (SNCPCH), que tinha como instrumento para entrada dos dados a Guia de Internação Hospitalar e, posteriormente, na década de 1980, o Sistema de Assistência Médico-Hospitalar da Previdência Social, tendo a AIH como instrumento pagador das internações hospitalares (Pepe, 2009). Em ambos os sistemas, se o prestador quisesse receber a remuneração pelo serviço prestado, a esfera pública necessariamente deveria remeter informações sobre a sua produção hospitalar. Contudo, nesse sistema, o processamento dos dados estava localizado em âmbito central e ele ainda não se encontrava descentralizado no prestador.

Em 1991, o sistema mudou de nomenclatura e passou a ser chamado de SIH e, desde então, as ideias de descentralização do processamento das informações para as SES, SMS e prestadores começaram a emergir. A Portaria n. 221, de 1999, determinou que essa descentralização fosse realizada em âmbito estadual e municipal. Assim, esta portaria caracteriza-se como uma pressão coercitiva exercida pelo Governo Federal, obrigando as SES e as SMS a processarem informações. Nesse momento, cabia ao prestador o repasse das $\mathrm{AIH}$ às secretarias de saúde e o sistema não havia sido descentralizado à sua competência.

A Portaria n. 510, de 2005, foi um marco para descentralização do SIHD no que se refere ao prestador. O E2 vivenciou esse processo e relatou que

“em 2006, quando descentralizaram o sistema de forma obrigatória, o prestador foi obrigado a começar a utilizar o sistema. Na hora, isso gerou uma preocupação geral, a gente se questionava: será que isso vai dar certo? Porque é muito prestador, é muita coisa, a gente achou que não ia dar certo, mas deu e dentro do prazo".

Mesmo com a descrença do entrevistado sobre a viabilidade da descentralização do sistema nesse nível, o processo ocorreu conforme estipulado pela portaria. Como a portaria possui caráter normativo e impositivo, isso evidencia que pressões coercitivas para utilização do SIHD foram impostas no ambiente organizacional, obrigando os prestadores a adequar-se às novas exigências do governo Federal a respeito do faturamento hospitalar.

Segundo o E4, é comum ao DATASUS que, antes de disseminar um SI de informação reformulado, faça um teste piloto em algum ente da federação, como pode ser apurado em seu relato:

"Normalmente, o DATASUS quando lança uma versão ele sempre faz um teste piloto em um estado, pode ser São Paulo, Paraná até Rio Grande do Sul. Eles escolhem um estado pra ver se o sistema vai dar certo, se precisa fazer alterações e daí, depois sim, lançam para o resto do Brasil a versão. Mas eu não sei te dizer onde foi feito [o teste piloto] do SIHD".

Esse teste piloto realizado pelo DATASUS configura uma forma de demonstrar aos outros estados os resultados do sistema, considerado como um caso bem-sucedido a ser implementado no restante do país, podendo gerar pressões miméticas no campo organizacional. Tal fato pôde permitir maior aceitação do sistema uma vez que apresentou suas potencialidades reduzindo o nível de incerteza. 
Retomando a questão da obrigatoriedade do sistema, além da responsabilidade do prestador de manusear e remeter os dados sobre o faturamento, as SES, igualmente, possuem a obrigação de remeter ao MS tais dados. Caso isso não ocorra, são atribuídas sanções, conforme pode ser verificado a seguir: "Suspensão da transferência do Bloco de financiamento da Média e Alta Complexidade nos casos da não alimentação por dois meses consecutivos ou três meses alternados" (Conselho Nacional de Secretários de Saúde [CONASS], 2011). O corte de recursos também é determinado pela Portaria n. 204, de 29 de janeiro de 2007, sendo este outro mecanismo coercitivo identificado para garantir, além da utilização do sistema, a remessa de informações ao governo federal.

\section{Resultados da Pesquisa}

Os três pilares da Teoria Institucional propostos por Scott (2008) serviram como base para a análise da influência de fatores institucionais na adoção de e-Serviços públicos por Instituições de Ensino Superior (IES), no estudo de Pereira, Macadar e Becker (2012). A influência na adoção do eServiço analisado ocorreu por pressões coercitivas, normativas e miméticas oriundas do contexto organizacional. As pressões institucionais em que foi pautado o estudo são: detenção de recursos e influência política no pilar regulatório; comportamento desejado, aceitação e valores no pilar normativo; e modelos bem-sucedidos e crenças comuns no pilar cultural-cognitivo. Tais pressões geraram as proposições de análise da pesquisa no sentido de que as IES dependentes de recursos e que necessitam manter sua posição no campo organizacional tendem a adotar e-Serviços públicos estabelecidos por instituições reconhecidas por influência de fatores institucionais (Pereira et al., 2012).

Para análise dos resultados da presente pesquisa, foi adaptado o modelo conceitual de Pereira, Macadar e Becker (2012), o que sugere uma relação entre fatores institucionais e a utilização de SIS pelas Secretarias Estaduais de Saúde (vide Figura 1).

\section{Campo Organizacional}

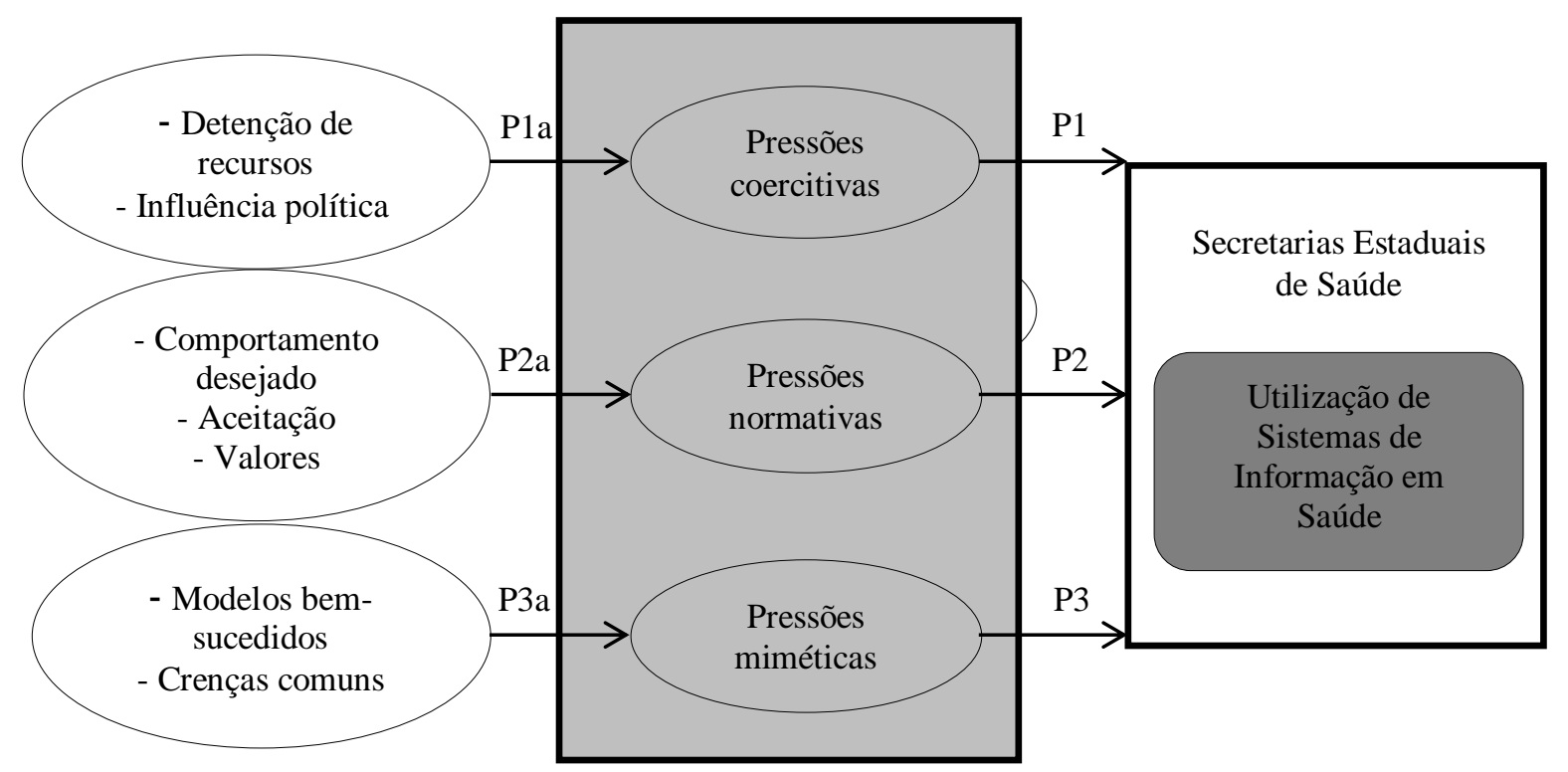

Figura 1. Modelo de Resultados da Pesquisa.

Fonte: Adaptado de Pereira, G. V., Macadar, M. A., \& Becker, G. V. (2012). Análise do uso do Portal de Periódicos da CAPES em uma IES sob Perspectiva Institucional. Análise (PUCRS), 23(1), 78-90.

Como resultado da pesquisa, tem-se as seguintes proposições teóricas, que são discutidas no decorrer desta seção. 
Proposição 1: as Secretarias Estaduais de Saúde dependentes de recursos e de influência política são coagidas a utilizar SIS estabelecidos por organizações dominantes do seu campo organizacional, por pressões coercitivas.

Proposição 1a: a detenção de recursos e a influência política por organizações dominantes geram pressões coercitivas no campo organizacional.

Proposição 2: as Secretarias Estaduais de Saúde que necessitam aceitação no seu campo organizacional tendem a utilizar SIS reconhecidos, por pressões normativas.

Proposição 2a: a existência de um comportamento desejado e uma tendência à profissionalização geram pressões normativas no campo organizacional.

Proposição 3: as Secretarias Estaduais de Saúde que necessitam reconhecimento tendem a utilizar SIS bem-sucedidos, por pressões miméticas.

Proposição 3a: a existência de modelos bem-sucedidos de SIS geram pressões miméticas no campo organizacional.

Assim, percebe-se que a detenção de recursos e influência política de organizações dominantes no campo organizacional influenciam as Secretarias Estaduais de Saúde na utilização dos SIS analisados, fator abordado nas proposições 1 e $1 \mathrm{a}$.

A respeito das pressões normativas existentes no âmbito das secretarias estaduais de saúde, citase o processo de profissionalização dos indivíduos que trabalham diretamente com os sistemas. Isso pode ser verificado a partir do entendimento de que o uso efetivo desses sistemas pressupõe um discernimento que vai além de como funciona a ferramenta, sendo necessário que esses indivíduos tenham conhecimento específico sobre a área da saúde e suas peculiaridades. Outra pressão normativa encontrada no campo relaciona-se com a utilização por ambos os sistemas do CID-10, um código internacional de doenças mundialmente difundido e reconhecido, fazendo com que as informações produzidas por esses sistemas tenham representatividade e possibilidade de comparação em âmbito internacional. Além disso, identifica-se que pressões normativas são referentes à profissionalização e à certificação verificadas na utilização desses SIS.

Dessa forma, apesar de identificado que a existência de um comportamento desejado e uma tendência à profissionalização geram pressões normativas no campo organizacional, corroborando a proposição 2a, a proposição 2 pode ser abordada apenas com relação aos SIS analisados, considerando que o DATASUS, por necessitar aceitação no seu campo organizacional, tende a utilizar em seus sistemas padrões internacionalmente reconhecidos, por meio de pressões normativas.

Neste estudo, as pressões miméticas podem ser consideradas aquelas oriundas do contexto internacional, em que organizações como a OMS e OPAS, que possuem como meta melhorar a situação de saúde da população mundial e das Américas, respectivamente, estavam sistematizando as informações em saúde em outros países. Na década de 1960, a OMS já publicava anuários sobre mortalidade, informações que até então não estavam informatizadas no país (Jorge et al., 2009). Dessa forma, considerando a situação de incertezas frente à gestão das informações em saúde no país, que ainda se encontravam desordenadas e eram provenientes de intervenções pontuais (Ministério da Saúde, 2009). Foi percebida a necessidade de adequar-se ao contexto internacional, uma vez que sistematizar suas informações poderia promover melhores condições de saúde à população. Com isso, percebe-se a existência de modelos bem-sucedidos de SIS que tendem a influenciar as Secretarias Estaduais de Saúde na utilização dos mesmos, proporcionando às instituições reconhecimento e legitimidade no campo organizacional, corroborando as proposições 3 e $3 \mathrm{a}$.

Enfim, considerando estas proposições, pode-se afirmar que foram dados os primeiros passos para o processo de institucionalização dos SIS em âmbito público no Brasil. 


\section{Considerações Finais}

Esta pesquisa propõe discussão atinente às influências institucionais na utilização de SIS por Secretarias Estaduais de Saúde. Foram apresentados os diferentes fatores institucionais que influenciam na utilização dos SIS, na forma de pressões coercitivas, miméticas e normativas no campo organizacional.

A análise sobre os fatores que influenciaram a utilização dos SIS, com o apoio da Teoria Institucional, possibilitou compreender os processos que ocorrem desde a concepção dos sistemas até a sua utilização extensiva pelas secretarias de saúde nos estados brasileiros. Foram detectadas fortes pressões miméticas e coercitivas na concepção e na utilização dos sistemas.

No SIM, pressões miméticas e coercitivas, oriundas de instituições internacionais como OMS e OPAS, foram decisivas para a concepção do sistema. Igualmente, pode-se citar a ausência nos estados de SI para organizar seus dados em saúde e a instituição de legislação (pressão coercitiva), assim, obrigando os entes federados a remeterem mensalmente seus dados, com penalidade atribuída ao descumprimento de tal normativa. Já no SIHD, descentralizado aos prestadores em 2006, viu-se a ação de forte pressão coercitiva por meio de portarias que obrigaram, primeiramente, as SES a responsabilizarem-se pela gestão desses dados e, depois, ao envio dos dados pelos prestadores de forma informatizada.

Além da adaptação do modelo conceitual trabalhado por Pereira et al. (2012) para outra dimensão de governo eletrônico (e-Administração) e outro contexto, este trabalho apresenta como contribuição teórica a análise de um fenômeno sob o foco multidisciplinar, apresentando convergências de análise provenientes de áreas distintas como Organizações, por meio da Teoria Institucional, e Sistemas de Informação e Saúde.

Como limitação da pesquisa, tem-se a descontinuidade dos profissionais que trabalham nas SES, fator que dificultou o recorte temporal do histórico da concepção desses sistemas. Sugere-se que estudos futuros investiguem outros SIS ofertados pelo DATASUS para verificar as influências ambientais na concepção desses sistemas. Como opção de futuras pesquisas, sugere-se a realização de estudos em secretarias municipais de saúde verificando as pressões existentes em âmbito local para adoção dessas tecnologias. No que se refere à gestão propriamente dita, investigações sobre a efetividade desses sistemas, para apoiar a tomada de decisão de gestores nos diferentes entes da federação, podem contribuir muito para os estudos organizacionais.

Por fim, destaca-se que o modelo de resultados, bem como as proposições teóricas provenientes deste, pode vir a ser utilizado na análise de fatores institucionais que influenciam não somente outros sistemas ofertados pelo DATASUS, mas também implementados em outras áreas da gestão pública. Eventualmente, um estudo correlacional que objetivasse modelar e testar estas proposições poderia ser empreendido por meio de uma pesquisa survey em âmbito nacional, assim, permitindo avaliar o nível de influência de cada um dos fatores institucionais.

\section{Referências}

Bansler, J. P., \& Havn, E. (2010). Pilot implementation of health information systems: Issues and challenges. International Journal of Medical Informatics, 79(9), 637-648. doi: 10.1016/j.ijmedinf.2010.05.004

Bardin, L. (2011). Análise de conteúdo. São Paulo: Edições 70.

Branco, M. A. F. (2006). Informação e saúde: uma ciência e suas políticas em uma nova era. Rio de Janeiro: Editora Fiocruz. 
Carvalho, D. M. T. (2009). Sistema de informações hospitalares do SUS - SIH-SUS. In A experiência brasileira em sistemas de informação em saúde/Ministério da Saúde, Organização PanAmericana da Saúde, Fundação Oswaldo Cruz (Vol. 2, pp. 49-70). Brasília: Editora MS.

Chiasson, M. W., \& Davidson, E. (2004). Pushing the contextual envelope: developing and diffusing IS theory for health information systems research. Information and Organization, 14(3), 155-188. doi: 10.1016/j.infoandorg.2004.02.001

Conselho Nacional de Secretários de Saúde. (2011). Sistema único de Saúde (SUS). (Vol.1, Coleção para entender a Gestão do SUS). Brasília: Autor.

Decreto n. 100, de 16 de abril de 1991. (1991). Institui a Fundação Nacional de Saúde e dá outras providências. Diário Oficial da União. Brasília, DF: Presidência da República. Casa Civil. Subchefia para assuntos jurídicos.

Deephouse, D. L., \& Suchman, M. (2008). Legitimacy in organizational institutionalism. In R. Greenwood, C. Oliver, K. Sahlin-Andersson, \& R. Suddaby (Eds.), The Sage handbook of organizational institutionalism (pp. 49-77). Thousand Oaks, CA: Sage.

DiMaggio, P. J., \& Powell, W. W. (1983). The iron cage revisited: institutional isomorphism and collective rationality in organizational fields. American Sociological Review, 48(2), 147-60. doi: 10.1016/S0742-3322(00)17011-1

DiMaggio, P. J., \& Powell, W. W. (1991). Introduction. In W. W. Powell \& P. J. DiMaggio (Eds.), The new institutionalism in organizational analysis (pp. 1-38.). Chicago: University of Chicago Press.

Diniz, E. H., Barbosa, A. F., Junqueira, A. R. B., \& Prado, O. (2009). O governo eletrônico no Brasil: perspectiva histórica a partir de um modelo estruturado de análise. Revista de Administração Pública, 43(1), 23-48. doi: 10.1590/S0034-76122009000100003

Fonseca, V. S. (2003). A abordagem institucional nos estudos organizacionais: bases conceituais e desenvolvimentos contemporâneos. In M. M. F. Vieira \& C. A. Carvalho (Orgs.), Organizações, instituições e poder no Brasil (pp. 47-66). São Paulo: FGV.

Frunkim, P., \& Galaskiewicz, J. (2004). Institutional isomorphism and public sector organizations. Journal of Public Administration and Theory, 14(3). 283-307. doi: 10.1093/jopart/muh028

Gibbs, G. (2009). Análise de dados qualitativos. Porto Alegre: Artmed.

Godoy, A. S. (1995). Pesquisa qualitativa: tipos fundamentais. Revista de Administração de Empresas, 35(3), 20-29. doi: 10.1590/S0034-75901995000300004

Haux, R. (2006). Health information systems - past, present, future. International Journal of Medical Informatics, 75(3/4), 268-281. doi: 10.1016/j.ijmedinf.2005.08.002

Heeks, R. (2006). Health information systems: failure, success and improvisation. International Journal of Medical Informatics, 75(2), 125-137. doi: 10.1016/j.ijmedinf.2005.07.024

Johnson, C. W. (2011). Identifying common problems in the acquisition and deployment of large-scale, safety-critical, software projects in the US and UK healthcare systems. Safety Science, 49(5), 735-745. doi: 10.1016/j.ssci.2010.12.003

Jones, C. R., Cardoso, R. B., Hüttner, E., Oliveira, H. W., Santos, M. A., Lopes, M. A. I., \& Russomano, T. (2012). Towards designing for equity: active citizen participation in eHealth. Transforming Government: People, Process and Policy, 6(4), 333-344. doi: 10.1108/17506161211267400

Jorge, M. H. P. Mello de, Laurenti, R., \& Gotlieb, S. L. D. (2009). O sistema de informações sobre mortalidade - SIM concepção, implantação e avaliação. In A experiência brasileira em sistemas 
de informação em saúde/Ministério da Saúde, Organização Pan-Americana da Saúde, Fundação Oswaldo Cruz (Vol. 2, pp. 71-108). Brasília: Editora MS.

Laia, M. M. de, Cunha, M. A. V. C, Nogueira, A. R. R., \& Mazzon, J. A. (2011). Electronic government policies in Brazil: context, ICT management and outcomes. Revista de Administração de Empresas, 51(1), 43-57. doi: 10.1590/S0034-75902011000100005

Machado-da-Silva, C. L., Fonseca, V. S. da, \& Crubellate, J. M. (2010). Estrutura, agência e interpretação: elementos para uma abordagem recursiva do processo de institucionalização [Edição Especial]. Revista de Administração Contemporânea, 14, 77-107. Recuperado de http://www.scielo.br/pdf/rac/v14nspe/a05v14ns.pdf. doi: 10.1590/S1415-65552010000600005

Machado-da-Silva, C. L., Guarido, E. R., Filho, Nascimento, M. R., \& Oliveira, P. T. (2003). Institucionalização da mudança na sociedade brasileira: o papel do formalismo. In M. M. F. Vieira \& C. A. Carvalho (Orgs.). Organizações, instituições e poder no Brasil (pp. 179-202). Rio de Janeiro: Editora FGV.

Mettler, T., \& Raptis, D. A. (2012). What constitutes the field of health information systems? Fostering a systematic framework and research agenda. Health Informatics Journal, 18(2), 147-156. doi: $10.1177 / 1460458212452496$

Meyer, J. W., \& Rowan, B. (1977). Institutionalized organizations: formal structures as myth and ceremony. The American Journal of Sociology, 83(2), 340-363.

Mignerat, M., \& Rivard, S. (2009). Positioning the institutional perspective in information systems research. Journal of Information Technology, 24(4), 369-391. doi:10.1057/jit.2009.13

Ministério da Saúde. (2009). A experiência brasileira em sistemas de informação em saúde/Ministério da Saúde, Organização Pan-Americana da Saúde, Fundação Oswaldo Cruz (Vol. 2). Brasília: Editora MS.

Pepe, V. E. (2009). Sistema de Informações Hospitalares do Sistema Único de Saúde (SIH-SUS). In Ministério da Saúde, Organização Pan-Americana da Saúde, Fundação Oswaldo Cruz. A experiência brasileira em sistemas de informação em saúde (Vol. 2, pp.65-85, Série B. Textos Básicos de Saúde). Brasília: Editora do Ministério da Saúde.

Pereira, G. V., Macadar, M. A., \& Becker, G. V. (2012). Análise do uso do portal de periódicos da CAPES em uma IES sob perspectiva institucional. Análise (PUCRS), 23(1), 78-90.

Portaria n. 221, de 24 de março de 1999. (1999). Determina que unidades hospitalares passem a informar ao Ministério da Saúde a ocorrência de eventos de internação hospitalar. Diário Oficial da União. Brasília, DF: Gabinete do Ministério da Saúde.

Portaria n. 474, de 31 de Agosto de 2000. (2000). Regulamenta a coleta de dados, fluxo e periodicidade de envio das informações sobre óbitos para o Sistema de Informações sobre Mortalidade - SIM. Diário Oficial da União. Brasília, DF: Autor. Ministério da Saúde. Fundação Nacional de Saúde.

Portaria n. 20, de 03 de outubro de 2003. (2003). Regulamenta a coleta de dados, fluxo e periodicidade de envio das informações sobre óbitos e nascidos vivos para os Sistemas de Informações sobre Mortalidade - SIM e sobre Nascidos Vivos -SINASC. Diário Oficial da União. Brasília, DF: Ministério da Saúde. Secretaria de Vigilância em Saúde.

Portaria n. 821, de 04 de maio de 2004. (2004). Determina a implantação gradativa da descentralização do processamento do Sistema de Informação Hospitalar - SIH. Diário Oficial da União. Brasília, DF: Gabinete do Ministério da Saúde. 
Portaria n. 510, de 30 de setembro de 2005. (2005). Estabelece a implantação do processamento descentralizado do Sistema de Informação Hospitalar - SIH. Diário Oficial da União. Brasília, DF: Ministério da Saúde. Secretaria de Atenção à Saúde.

Portaria n. 204, de 29 de janeiro de 2007. (2007). Regulamenta o financiamento e a transferência dos recursos federais para as ações e os serviços de saúde. Diário Oficial da União. Brasília, DF: Gabinete do Ministério da Saúde.

Ranson, S., Hinings, B., \& Greenwood, R. (1980). The structuring of organizational structures. Administrative Science Quarterly, 25(1), 1-17.

Ronchi, E., \& Senne, F. (2014). Melhores sistemas de medição são cruciais para concretizar todo o potencial das TIC no setor de Saúde. In A. F. Barbosa (Coord.), TIC Saúde 2013: [livro eletrônico]: pesquisa sobre o uso das tecnologias de informação e comunicação nos estabelecimentos de saúde brasileiros (pp. 59-66). São Paulo: Comitê Gestor de Internet no Brasil. Recuperado de http://cetic.br/media/docs/publicacoes/2/tic-saude-2013.pdf

Scott, W. R. (2008). Institutions and organizations (3rd ed.). Thousand Oaks, CA: Sage Publications.

Selznick, P. (1957). Leadership in administration. Evanston: Northwestern University Press.

Spil, T. A. M., LeRouge, C., Trimmer, K., \& Wiggins, C. (2009). IT adoption and evaluation in healthcare: evolutions and insights in theory, methodology and practice. International Journal of Healthcare Information Systems and Informatics, 4(3), 69-96. doi: 10.4018/jhisi.2009070105

Souza, M., Cunha, M. X. C., Jr., \& Dornelas, J. S. (2011, maio). Examinando as dimensões burocráticas dos sistemas de informação em saúde pública a partir das lentes teóricas de Max Weber. Anais do Encontro de Administração da Informação. Porto Alegre, Brasil, 3.

Suchman, M. C. (1995). Managing legitimacy: strategic and institutional approaches. Academy of Management Review, 20(3), 571-610. doi: 10.5465/AMR.1995.9508080331

Tolbert, P. S., \& Zucker, L. G. (1999). A institucionalização da teoria institucional. In S. R. Clegg, C. Hardy, \& W. R. Nord (Orgs.), Handbook de estudos organizacionais (Vol. 1, Cap. 6, pp. 196219). São Paulo: Atlas.

Vidor, A. C., Fisher, P. D., \& Bordin, R. (2011). Utilização dos sistemas de informação em saúde em municípios gaúchos de pequeno porte. Revista de Saúde Pública, 45(1), 24-30. doi: 10.1590/S0034-89102011000100003

World Health Organization. (n.d.). International classification of diseases (ICD). Recuperado de http://www.who.int/classifications/icd/en/

World Health Organization. (2004). Developing health management information systems: a practical for developing countries. Manila: Regional Office for the Western Pacific. 